\title{
T-duality, non-geometry and Lie algebroids in heterotic double field theory
}

\section{Ralph Blumenhagen and Rui Sun}

Max-Planck-Institut für Physik (Werner-Heisenberg-Institut),

Föhringer Ring 6, 80805 München, Germany

E-mail: blumenha@mpp.mpg.de, sunrui@mpp.mpg.de

AbStract: A number of issues in heterotic double field theory are studied. This includes the analysis of the T-dual configurations of a flat constant gauge flux background, which turn out to be non-geometric. Performing a field redefinition to a non-geometric frame, these T-duals take a very simple form reminiscent of the constant $Q$ - and $R$-flux backgrounds. In addition, it is shown how the analysis of arXiv:1304.2784 generalizes to heterotic generalized geometry. For every field redefinition specified by an $O(D, D+n)$ transformation, the structure of the resulting supergravity action is governed by the differential geometry of a corresponding Lie algebroid.

KEYwORDS: Flux compactifications, Superstrings and Heterotic Strings

ARXiv EPRINT: 1411.3167 


\section{Contents}

1 Introduction 1

2 Review of heterotic DFT 4

2.1 The generalized metric 4

2.2 Generalized vielbeins in heterotic DFT 5

3 Non-geometric backgrounds of heterotic DFT $\quad 7$

3.1 T-duality of a constant gauge flux background $\quad 7$

$\begin{array}{ll}3.2 & \text { The fluxes of heterotic DFT } \\ \end{array}$

3.3 Comment on $R$-flux and non-associativity 12

$\begin{array}{ll}3.4 \text { Comment on S-duality } & 12\end{array}$

4 A Lie algebroid for heterotic field redefinitions $\quad \mathbf{1 3}$

$\begin{array}{lll}4.1 & O(D, D+n) \text {-induced field redefinition } & 13\end{array}$

$\begin{array}{ll}4.2 \text { The redefined heterotic action } & 16\end{array}$

$\begin{array}{ll}4.3 \text { The non-geometric frame } & 17\end{array}$

$\begin{array}{llr}5 & \text { Conclusion } & 18\end{array}$

$\begin{array}{ll}\text { A The Buscher rules derived from heterotic DFT } & 19\end{array}$

$\begin{array}{ll}\text { B Non-holonomic fluxes for heterotic DFT } & 20\end{array}$

$\begin{array}{ll}\text { C Lie algebroids } & 21\end{array}$

\section{Introduction}

The description and understanding of non-geometric string backgrounds have been under investigation during the last years. As with many other developments in string theory, the exploration of the consequences of T-duality has been a good guide in this respect [1-4]. The classic example is to perform successive T-dualities via the Buscher rules [5, 6] applied to a flat closed string background with constant $H$-flux [3]. This led to the chain of flux backgrounds with fluxes $H_{a b c} \rightarrow F_{b c}^{a} \rightarrow Q_{c}{ }^{a b} \rightarrow R^{a b c}$ where it was shown that the $Q$-flux background is non-geometric globally and the $R$-flux background even locally. It was shown that these non-geometric backgrounds take a very simple form, when expressed not in the geometric frame $\left(g_{i j}, B_{i j}\right)$ but in a so-called non-geometric frame $\left(\tilde{g}_{i j}, \beta^{i j}\right)$, where the new metric and the bi-vector are related to the geometric frame via a field redefinition.

In order to properly describe such backgrounds, one needs to go beyond the usual effective supergravity description of string theory. In this respect, two approaches were 
followed. The first one is generalized geometry [7-10], where one extends the tangent bundle of a manifold such that diffeomorphisms and $B$-field gauge transformations can be described in a single geometric framework. Concretely, the metric and the Kalb-Ramond field are unified in a generalized metric on the bundle $T \oplus T^{*}$. A more ambitious approach is to develop a theory which is manifestly invariant under T-duality. This led to double field theory (DFT), where not only the bundle but also the coordinates themselves are extended to a doubled space by introducing winding coordinates. A first approach followed a framelike formulation $[11,12]$ which was further worked out in $[13,14]$. Later, using string field theory, an equivalent generalized metric formulation was found [15-17]. However, DFT not only features a global $O(D, D)$ symmetry but also the local symmetries, due to the winding dependence, are enhanced. For recent reviews of DFT see [18-20].

Whether generalized geometry allows for a description of non-geometric backgrounds has been investigated in a series of papers. In particular, in [21-23] the question has been asked what form the usual supergravity action takes in the non-geometric frame variables and whether this action might already be sufficient for the global description of nongeometric backgrounds. This led to the definition of so-called $\beta$-supergravity. In $[24,25]$ the general structure of such $O(D, D)$ induced field redefinitions was clarified in the framework of generalized geometry. The two main results were that for every such field redefinition, one can associate a corresponding Lie algebroid so that the redefined supergravity action is governed by the differential geometry of that Lie algebroid. It turned out that in each patch this provides are good description of the background, but that the transition functions needed for the global description in general are not part of the local symmetries of generalized geometry. ${ }^{1}$

On the contrary, due to the existence of extra local symmetries in DFT, i.e. the generalized diffeomorphisms, the latter admits a global description of the $Q$ and $R$-flux backgrounds. The non-geometry shows up for the $Q$-flux background in a winding dependence of the transition function between two patches and for the $R$-flux in an explicit winding dependence of the background field itself.

A natural generalization of bosonic DFT is heterotic DFT [11, 12, 27, 28], where the latter also includes the gauge fields present in the heterotic string. In generalized geometry the heterotic string was also discussed in [29-31](see also [32, 33]). For abelian gauge fields this generalization is formally straightforward extending the global symmetry group from $O(D, D)$ to $O(D, D+n)$. For every gauge field $A^{\alpha}$ a new coordinate $y^{\alpha}$ is introduced, thus extending also the generalized metric so that it includes the gauge fields. The main relations of DFT remain unchanged so that the action still has the same form as for bosonic DFT, but just for the extended generalized metric. This abelian heterotic DFT can be gauged which also allows the description of non-abelian gauge groups $[28,34]$. However, in this process the part of the global symmetry group is broken to $O(D, D)$.

It was observed in [35] that, in contrast to bosonic DFT, the action of T-duality gives the Buscher rules including $\alpha^{\prime}$ corrections. In the same work, a suggestion has been made how heterotic DFT can be further generalized to also accommodate the leading order

\footnotetext{
${ }^{1}$ For possible exceptions see [26].
} 
gravitational $\alpha^{\prime}$ corrections, including e.g. the well known Chern-Simons terms involving the spin-connection. There has been quite some interest recently on how to incorporate such $\alpha^{\prime}$ corrections in the framework of generalized geometry [36, 37] and DFT [35, 38-40].

In this paper, in some sense, we take a step back from these more formal developments and investigate some comparably simple questions which, as we think, are nevertheless important to clarify. For instance, to our knowledge, it is not clear what the heterotic Tdual of a constant gauge flux background is. The same question for the S-dual background of a type I string led to the discovery of $D$-branes and $O$-planes. Indeed the T-dual of a $D 9$-brane carrying a constant gauge flux in type I is the type I' string with a $D 8$ brane intersecting the $O 8$-plane at an angles. In the heterotic case there are no 8-branes so what is the T-dual? Not unrelated, one can ask whether for heterotic DFT, there exist an analogous chain of T-dual fluxes as for bosonic DFT. Of course, the $H_{i j k} \rightarrow F_{i j}{ }^{k} \rightarrow Q_{i}{ }^{j k} \rightarrow R^{i j k}$ chain will still exist, but what about a similar chain starting with an abelian constant gauge flux $G_{i j} \rightarrow \ldots$ ? Does it also give rise to new types of non-geometric fluxes? Clearly, these are questions one can now approach in the framework of heterotic DFT.

We will find that indeed after one T-duality, one gets a non-geometric gauge flux background that is in many ways analogous to the $Q$-flux background. It is locally still geometric and the non-geometry appears in the transition functions in the sense that there appears a dependence on a winding coordinate. Moreover, also for heterotic DFT one can perform a field redefinition to a non-geometric frame in which the fundamental fields are a dual metric $\tilde{g}_{i j}$, a bi-vector $\beta^{i j}$ and a gauge one-vector $\tilde{A}^{i}$. We will see that one indeed gets a chain of fluxes $G_{i j} \rightarrow J^{i}{ }_{j} \rightarrow \tilde{G}^{i j}$, where the latter two are non-geometric. One can trace back that, in this case, the non-geometry arises due to the $\alpha^{\prime}$ corrections to the T-duality rules [41, 42].

Having realized that a field redefinition can be important for the description of nongeometric backgrounds, we can ask how the analysis of [25] generalizes to the heterotic case. Can the effect of an $O(D, D+n)$ induced field redefinition still be described by the differential geometry of a corresponding Lie algebroid? We will see that this is indeed possible and explicitly present the corrections due to the existence of the gauge field. As for the original version, the local symmetries of the redefined action are only the redefined versions of diffeomorphisms, $B$-field and $A$-field gauge transformation. This implies that a single such action cannot globally describe non-geometric backgrounds, which need winding coordinates to appear either in the transition functions ( $Q$-flux) or in the background itself ( $R$-flux).

This paper is organized as follows: in section 2 we briefly introduce the setup for heterotic DFT and the basics we need for our discussion. In section 3 we will perform in detail successive T-dualities on a toroidal constant gauge flux background and show how a field redefinition to a non-geometric frame simplifies the description of the T-dual backgrounds. We derive explicitly the form of the relevant heterotic fluxes and comment on the consequences for a potential non-associativity and for S-duality to the type I string. In section 4 we discuss the $O(D, D+n)$ induced field redefinitions and identify the corresponding Lie algebroid. In particular, we present the form of the action in the previously introduced non-geometric frame. 


\section{Review of heterotic DFT}

In this section we briefly review the bosonic sector of heterotic DFT, where we focus on those features which are important in the remainder of this note. The bosonic sector of heterotic DFT with abelian gauge fields is a straightforward generalization of bosonic DFT [28]. This is expected, as abelian gauge fields appear by dimensional reduction of gravity theories.

The low-energy effective action of the massless bosonic sector for the heterotic string is described by the action

$$
\mathcal{S}=\int d x \sqrt{g} e^{-2 \phi}\left(R+4(\partial \phi)^{2}-\frac{1}{12} H^{i j k} H_{i j k}-\frac{1}{4} G^{i j}{ }_{\alpha} G_{i j}{ }^{\alpha}\right)
$$

in which the field strength of the non-abelian gauge fields is defined as

$$
G_{i j}{ }^{\alpha}=\partial_{i} A_{j}{ }^{\alpha}-\partial_{j} A_{i}{ }^{\alpha}+g_{0}\left[A_{i}, A_{j}\right]^{\alpha}
$$

and the strength of the Kalb-Ramond field is modified by the Chern-Simons three-form,

$$
H_{i j k}=3\left(\partial_{[i} B_{j k]}-\kappa_{\alpha \beta} A_{[i}^{\alpha} \partial_{j} A_{k]}^{\beta}-\frac{1}{3} g_{0} \kappa_{\alpha \beta} A_{[i}{ }^{\alpha}\left[A_{j}, A_{k]}\right]^{\beta}\right) .
$$

Here $\kappa_{\alpha \beta}$ denotes the Cartan-Killing metric of the gauge group. In the abelian case, this is simply the unit matrix, $\kappa_{\alpha \beta}=\delta_{\alpha \beta}$. Note that the order in $\alpha^{\prime}$ can be made visible by scaling $A_{i}{ }^{\alpha} \rightarrow \sqrt{\alpha^{\prime}} A_{i}{ }^{\alpha}$. From now on we consider abelian gauge fields. Moreover, the higher derivative correction of $H$ due to the gravitational Chern-Simons form will be not considered throughout this paper.

\subsection{The generalized metric}

In the DFT formulation of the abelian heterotic sting [28], for each gauge field $A^{\alpha}$ one introduces a new coordinate $y^{\alpha}$ so that the entire DFT lives on a $2 D+n$ dimensional space with coordinates

$$
X^{M}=\left(\tilde{x}_{i}, x^{i}, y^{\alpha}\right)
$$

The global symmetry group is enhanced from $O(D, D)$ to $O(D, D+n)$, the T-duality group of the heterotic string. The doubled coordinates $X^{M}$ on a torus transform as an $O(D, D+n)$ vector

$$
X^{\prime M}=h^{M}{ }_{N} X^{N}, \quad h \in O(D, D+n) .
$$

As in bosonic DFT, one introduces an $O(D, D+n)$ invariant metric

$$
\eta_{M N}=\left(\begin{array}{ccc}
0 & \delta^{i}{ }_{j} & 0 \\
\delta_{i}{ }^{j} & 0 & 0 \\
0 & 0 & \delta_{\alpha \beta}
\end{array}\right)
$$

satisfying

$$
\eta^{M N}=h_{P}^{M} h_{Q}^{N} \eta^{P Q}
$$


This $O(D, D+n)$ metric is used to pull up and down capital indices like $M$. Accordingly, the generalized derivatives and gauge parameters are given as

$$
\partial_{M}=\left(\tilde{\partial}^{i}, \partial_{i}, \partial_{\alpha}\right), \quad \xi^{M}=\left(\tilde{\xi}_{i}, \xi^{i}, \Lambda^{\alpha}\right) .
$$

As shown in [28], one can proceed along the lines of bosonic DFT and introduce a generalized Lie derivative and a C-bracket. Then, closure of the algebra is guaranteed, if one introduces the heterotic strong constraint

$$
\partial_{M} f \partial^{M} g=\tilde{\partial}^{i} f \partial_{i} g+\partial_{i} f \tilde{\partial}^{i} g+\partial_{\alpha} f \partial^{\alpha} g=0
$$

where $f$ and $g$ are arbitrary fields and gauge parameters. This means that the heterotic level-matching condition

$$
\partial_{M} \partial^{M} f=2 \tilde{\partial}^{i} \partial_{i} f+\partial_{\alpha} \partial^{\alpha} f=0
$$

also has to hold for products of fields and implies that locally there exist an $O(D, D+n)$ transformation rotating the coordinates into a frame in which the fields only depend on the normal coordinates $x^{i}$.

The heterotic DFT action can be expressed in terms of a generalized metric and an $O(D, D+n)$ invariant dilaton $d$ defined by $e^{-2 d}=\sqrt{g} e^{-2 \phi}$. The metric $\mathcal{H}^{M N}$ transforms covariantly under $O(D, D+n)$

$$
\mathcal{H}^{\prime M N}\left(X^{\prime}\right)=h^{M}{ }_{P} h^{N}{ }_{Q} \mathcal{H}^{P Q}(X)
$$

and is parameterized in terms of the metric $g_{i j}$, the Kalb-Ramond field $B_{i j}$ and the gauge fields $A_{i}{ }^{\alpha}$ as

$$
\mathcal{H}_{M N}=\left(\begin{array}{ccc}
g^{i j} & -g^{i k} C_{k j} & -g^{i k} A_{k \beta} \\
-g^{j k} C_{k i} & g_{i j}+C_{k i} g^{k l} C_{l j}+A_{i}^{\gamma} A_{j \gamma} & C_{k i} g^{k l} A_{l \beta}+A_{i \beta} \\
-g^{j k} A_{k \alpha} & C_{k j} g^{k l} A_{l \alpha}+A_{j \alpha} & \delta_{\alpha \beta}+A_{k \alpha} g^{k l} A_{l \beta}
\end{array}\right)
$$

where only the combination

$$
C_{i j}=B_{i j}+\frac{1}{2} A_{i}^{\alpha} A_{j \alpha}
$$

appears. Note that $C_{i j}$ splits into a symmetric and an antisymmetric part as

$$
C_{(i j)}=\frac{1}{2} A_{i}^{\alpha} A_{j \alpha}, \quad C_{[i j]}=B_{i j} .
$$

Written in terms of the generalized metric (2.12), the form of the heterotic DFT action is identical to the action of bosonic DFT.

\subsection{Generalized vielbeins in heterotic DFT}

In analogy to bosonic DFT, one can also introduce a generalized vielbein $E^{A}{ }_{M}$ so that

$$
\mathcal{H}_{M N}=E^{A}{ }_{M} S_{A B} E^{B}{ }_{N}
$$


with the constant generalized metric

$$
S_{A B}=\left(\begin{array}{ccc}
s^{a b} & 0 & 0 \\
0 & s_{a b} & 0 \\
0 & 0 & s_{\alpha \beta}
\end{array}\right)
$$

and $s_{a b}=\operatorname{diag}(-,+, \ldots,+), s_{\alpha \beta}=\operatorname{diag}(+, \ldots,+)$. One finds ${ }^{2}$

$$
E^{A}{ }_{M}=\left(\begin{array}{ccc}
e_{a}{ }^{i}-e_{a}{ }^{k} C_{k i} & -e_{a}{ }^{k} A_{k \beta} \\
0 & e^{a}{ }_{i} & 0 \\
0 & A_{i}{ }^{\alpha} & \delta^{\alpha}{ }_{\beta}
\end{array}\right), \quad E_{A}{ }^{M}=\left(\begin{array}{ccc}
e^{a}{ }_{i} & 0 & 0 \\
-e_{a}{ }^{k} C_{k i} & e_{a}{ }^{i} & -e_{a}{ }^{k} A_{k}{ }^{\beta} \\
A_{i \alpha} & 0 & \delta_{\alpha}{ }^{\beta}
\end{array}\right)
$$

which also satisfies

$$
\eta_{M N}=E^{A}{ }_{M} E_{A N} .
$$

Now one defines the generalized derivative as

$$
D_{A}=E_{A}^{M} D_{M}=\left(\tilde{D}^{a}, D_{a}, D_{\alpha}\right)
$$

leading in components to

$$
\begin{aligned}
& \tilde{D}^{a}=\tilde{\partial}^{a} \\
& D_{a}=\partial_{a}-B_{a i} \tilde{\partial}^{i}-\frac{1}{2} A_{a}^{\alpha} A_{i \alpha} \tilde{\partial}^{i}-A_{a}^{\gamma} \partial_{\gamma} \\
& D_{\alpha}=\partial_{\alpha}+A_{i \alpha} \tilde{\partial}^{i} .
\end{aligned}
$$

Introducing the generalized Weitzenböck connection as

$$
\Omega_{A B C}=D_{A} E_{B}{ }^{N} E_{C N}
$$

the generalized fluxes of heterotic DFT are defined as

$$
\mathcal{F}_{A B C}=E_{C M} \mathcal{L}_{E_{A}} E_{B}{ }^{M}=\Omega_{A B C}+\Omega_{C A B}-\Omega_{B A C} .
$$

In a holonomic basis one finds e.g. the three-form flux

$$
H_{i j k}=-3\left(\partial_{[\underline{i}} B_{\underline{j k}]}-\delta_{\alpha \beta} A_{[\underline{i}}^{\alpha} \partial_{\underline{j}} A_{\underline{k}]}^{\beta}\right)
$$

which is precisely the field strength of the Kalb-Ramond field modified by the Chern-Simons three-form (2.3). In section 3.2, all these generalized fluxes will be evaluated more explicitly.

\footnotetext{
${ }^{2}$ Note that compared to [14], we have two different signs in the definition of the vielbein. This is because we want to be consistent with the generalized metric as defined in [28].
} 


\section{Non-geometric backgrounds of heterotic DFT}

In this section we will use the formalism of heterotic DFT to determine the T-dual of a heterotic string compactified on a two-torus $T^{2}$ with a constant gauge flux turned on. This is analogous to the configuration of $T^{3}$ with constant $H$ flux for bosonic string theory [3, 43]. In the latter case, this was the prototype example to detect after two T-dualities the possibility of a non-geometric $Q$-flux background. Applying a third T-duality led to the conjecture for the existence of an $R$-flux background. Applying these T-dualities in the framework of DFT, the non-geometry shows up in the appearance of winding coordinates in the transitions functions for the $Q$-flux and in the background itself for the $R$-flux. Thus, in this sense a $Q$-flux background is locally geometric but not globally, whereas an $R$-flux background is non-geometric even locally.

\subsection{T-duality of a constant gauge flux background}

Recall that under a global $h \in O(D, D+n)$ transformation the coordinates and the generalized metric behave as

$$
H^{\prime}=h^{t} H h, \quad X^{\prime}=h X, \quad \partial^{\prime}=\left(h^{t}\right)^{-1} \partial .
$$

Now, we consider a torus $T^{2}$ with a flat metric $g_{i j}=\delta_{i j}$, vanishing Kalb-Ramond field $B$ and a constant abelian gauge flux $G_{i j}$. For the corresponding single gauge potential $A^{(1)}=A$ we choose

$$
A_{1}=f y, \quad A_{2}=0 .
$$

This gives the field strength

$$
G_{12}=-\left(\partial_{1} A_{2}-\partial_{2} A_{1}\right)=f .
$$

On the 2 -torus the coordinates are periodically identified by $(x, y) \sim(x+2 \pi, y) \sim(x, y+$ $2 \pi)$. Let us introduce a patch $P$ for $0 \leq y<2 \pi$ and patch $Q$ for $0<y \leq 2 \pi$. In the patch $P$ we have $A_{1}^{(P)}=f y$ while in the patch $Q$ the gauge field is $A_{1}^{(Q)}=f(y-2 \pi)$. These two patches can be glued smoothly together by a gauge transformation $A_{1}^{(Q)}=A_{1}^{(P)}+\partial_{1} \lambda^{(P Q)}$ with

$$
\lambda^{(P Q)}=-2 \pi f x .
$$

The generalized metric for this background in patch $P$ takes the form

$$
\mathcal{H}_{M N}^{(P)}=\left(\begin{array}{cr|cr|c}
1 & 0 & -\frac{(f y)^{2}}{2} & 0 & -(f y) \\
0 & 1 & 0 & 0 & 0 \\
\hline-\frac{(f y)^{2}}{2} & 0 & 1+(f y)^{2}+\frac{(f y)^{4}}{4} & 0 & (f y)+\frac{(f y)^{3}}{2} \\
0 & 0 & 0 & 1 & 0 \\
\hline-(f y) & 0 & (f y)+\frac{(f y)^{3}}{2} & 0 & 1+(f y)^{2}
\end{array}\right) .
$$

The transition to patch $Q$ is given by conjugation with an appropriate $O(D, D+n)$ matrix $\mathcal{T}_{(P Q)}$, i.e.

$$
\mathcal{H}^{(Q)}=\mathcal{T}_{(P Q)}^{T} \mathcal{H}^{(P)} \mathcal{T}_{(P Q)}
$$


which in our case takes the form

$$
\mathcal{T}_{(P Q)}=\left(\begin{array}{cc|cc|c}
1 & 0 & -\frac{(2 \pi f)^{2}}{2} & 0 & 2 \pi f \\
0 & 1 & 0 & 0 & 0 \\
\hline 0 & 0 & 1 & 0 & 0 \\
0 & 0 & 0 & 1 & 0 \\
\hline 0 & 0 & -2 \pi f & 0 & 1
\end{array}\right) .
$$

In analogy to generalized geometry such a matrix might be called an " $A$-transform". Note that this is consistent with the discussion in [23], where the transition matrix was calculated via the vielbeins in the two patches as $\mathcal{T}_{(P Q)}=E_{(P)}^{-1} E_{(Q)}$.

Now, we apply a T-duality in the $x$-direction, which in heterotic DFT can be implement by conjugation $\mathcal{H}^{\prime}=\mathcal{T}_{1}^{T} \mathcal{H} \mathcal{T}_{1}$ with the special $O(2,3)$ transformation

$$
\mathcal{T}_{1}=\left(\begin{array}{ll|ll|l}
0 & 0 & 1 & 0 & 0 \\
0 & 1 & 0 & 0 & 0 \\
\hline 1 & 0 & 0 & 0 & 0 \\
0 & 0 & 0 & 1 & 0 \\
\hline 0 & 0 & 0 & 0 & 1
\end{array}\right) .
$$

The upper $4 \times 4$ dimensional part of the metric is the same as the T-duality transformation for bosonic DFT. Thus, we obtain in patch $P$

$$
\mathcal{H}^{\prime(P)}=\left(\begin{array}{cc|cc|c}
1+(f y)^{2}+\frac{(f y)^{4}}{4} & 0 & -\frac{(f y)^{2}}{2} & 0 & (f y)+\frac{(f y)^{3}}{2} \\
0 & 1 & 0 & 0 & 0 \\
\hline-\frac{(f y)^{2}}{2} & 0 & 1 & 0 & -(f y) \\
0 & 0 & 0 & 1 & 0 \\
\hline(f y)+\frac{(f y)^{3}}{2} & 0 & -(f y) & 0 & 1+(f y)^{2}
\end{array}\right)
$$

from which one can directly read off the new metric, Kalb-Ramond field and the gauge field as

$$
g^{\prime(P)}=\left(\begin{array}{cc}
\frac{1}{1+(f y)^{2}+\frac{(f y)^{4}}{4}} & 0 \\
0 & 1
\end{array}\right), \quad B^{\prime(P)}=0, \quad A^{\prime(P)}=\left(\begin{array}{c}
-\frac{(f y)}{1+\frac{(f y)^{2}}{2}} \\
0
\end{array}\right) .
$$

Note that after one T-duality one still gets a metric and a gauge field, where, as in the $Q$-flux background, there appears a non-trivial functional dependence in the denominators. Moreover, these results are consistent with the $\alpha^{\prime}$ corrected Buscher rules for a T-duality along a single direction for the heterotic string given in [42]. In fact, as shown in appendix A these T-duality rules following from the heterotic DFT construction are precisely the ones derived before from the Buscher rules of heterotic string theory. 
The new transition matrix to patch $Q$ is given by

$$
\mathcal{T}_{(P Q)}^{\prime}=\mathcal{T}_{1}^{T} \mathcal{T}_{(P Q)} \mathcal{T}_{1}=\left(\begin{array}{cc|cc|c}
1 & 0 & 0 & 0 & 0 \\
0 & 1 & 0 & 0 & 0 \\
\hline-\frac{(2 \pi f)^{2}}{2} & 0 & 1 & 0 & 2 \pi f \\
0 & 0 & 0 & 1 & 0 \\
\hline-2 \pi f & 0 & 0 & 0 & 1
\end{array}\right)
$$

which is not any longer a usual $A$-transform, i.e. a gauge transformation. This observation and the appearance of strange denominators already indicates that we are dealing here rather with a non-geometric background (like the $Q$-flux for bosonic DFT).

In analogy to bosonic DFT, one can introduce a field redefinition so that the generalized metric is parameterized by a new metric $\tilde{g}_{i j}$, a bi-vector $\tilde{C}^{i j}$ and a (one-)vector $\tilde{A}^{i}$ as

$$
\mathcal{H}_{M N}=\left(\begin{array}{ccc}
\tilde{g}^{i j}+\tilde{C}^{k i} \tilde{g}_{k l} \tilde{C}^{l j}+\tilde{A}^{i}{ }_{\gamma} \tilde{A}^{j \gamma}-\tilde{g}_{j k} \tilde{C}^{k i} & \tilde{C}^{k i} \tilde{g}_{k l} \tilde{A}_{\beta}+\tilde{A}^{i}{ }_{\beta} \\
-\tilde{g}_{i k} \tilde{C}^{k j} & \tilde{g}_{i j} & -\tilde{g}_{i k} \tilde{A}^{k}{ }_{\beta} \\
\tilde{C}^{k j} \tilde{g}_{k l} \tilde{A}_{\alpha}^{l}+\tilde{A}^{j}{ }_{\alpha} & -\tilde{g}_{j k} \tilde{A}^{k}{ }_{\alpha} \delta_{\alpha \beta}+\tilde{A}^{k}{ }_{\alpha} \tilde{g}_{k l} \tilde{A}_{\beta}^{l}{ }_{\beta}
\end{array}\right) .
$$

Here $\tilde{C}^{i j}=\beta^{i j}+\frac{1}{2} \tilde{A}_{\alpha}^{i} \tilde{A}^{j \alpha}$, where $\beta^{i j}$ is the antisymmetric bi-vector appearing also in bosonic DFT. The generalized vielbein reads in this case

$$
E^{A}{ }_{M}=\left(\begin{array}{ccc}
\tilde{e}_{a}{ }^{i} & 0 & 0 \\
-\tilde{e}^{a}{ }_{k} \tilde{C}^{k i} & \tilde{e}^{a}{ }_{i} & -\tilde{e}^{a}{ }_{k} \tilde{A}^{k}{ }_{\beta} \\
\tilde{A}^{i \alpha} & 0 & \delta^{\alpha}{ }_{\beta}
\end{array}\right)
$$

Comparing (3.9) with the form of the generalized metric in the so-called non-geometric frame (3.12), one can read off

$$
\tilde{g}^{(P)}=\left(\begin{array}{ll}
1 & 0 \\
0 & 1
\end{array}\right), \quad \tilde{A}^{\prime(P)}=\left(\begin{array}{c}
f y \\
0
\end{array}\right)
$$

with $\beta^{i j}=0$. This shows that in this frame the T-dual configuration takes a very simple form. Moreover, using (3.11) one can also find the metric and the one-vector in patch $\mathrm{Q}$

$$
\tilde{g}^{\prime(Q)}=\left(\begin{array}{ll}
1 & 0 \\
0 & 1
\end{array}\right), \quad \tilde{A}^{\prime(Q)}=\left(\begin{array}{c}
f(y-2 \pi) \\
0
\end{array}\right),
$$

Since the T-duality also changes $x \rightarrow \tilde{x}$ in the gauge transformation (3.4), the "gauge" transformation connecting the one-vectors in patch $P$ and $Q$ becomes

$$
\tilde{A}^{\prime 1(Q)}=\tilde{A}^{\prime 1(P)}+\tilde{\partial}^{1} \tilde{\lambda}^{(P Q)} \quad \text { with } \quad \tilde{\lambda}^{(P Q)}=-2 \pi f \tilde{x} .
$$

Note, that the transition function in this non-geometric frame contains a winding coordinate so that indeed this T-dual background is globally non-geometric, very similar to the $Q$-flux background for bosonic DFT. The only difference is that the latter requires 
a T-duality in two-directions in order to generate it from a constant $H$-flux background. Finally, the new flux in this T-dual background should be

$$
J^{1}{ }_{2}=-\partial_{2} \tilde{A}^{1}=-f .
$$

Applying to this configuration another T-duality in the $y$ direction only changes $y \rightarrow \tilde{y}$ in the generalized metric (3.9), so that in the non-geometric frame one obtains

$$
\tilde{g}^{\prime \prime(P)}=\left(\begin{array}{ll}
1 & 0 \\
0 & 1
\end{array}\right), \quad \tilde{A}^{\prime \prime(P)}=\left(\begin{array}{c}
f \tilde{y} \\
0
\end{array}\right),
$$

and similarly in patch $Q$. Therefore, like the $R$-flux background, this configuration is already locally non-geometric, characterized by a non-geometric flux

$$
\tilde{G}^{12}=-\left(\tilde{\partial}^{1} \tilde{A}^{2}-\tilde{\partial}^{2} \tilde{A}^{1}\right)=f .
$$

Of course, at this stage the form of the new non-geometric fluxes $J^{i}{ }_{j}$ and $\tilde{G}^{i j}$ is just a guess. In the following subsection, we derive the complete form of this new kind of fluxes from the vielbein (3.13).

\subsection{The fluxes of heterotic DFT}

In this section we derive the general form of the components of the heterotic fluxes

$$
\mathcal{F}_{A B C}=E_{C M} \mathcal{L}_{E_{A}} E_{B}{ }^{M}=\Omega_{A B C}+\Omega_{C A B}-\Omega_{B A C} .
$$

In order to treat geometric and non-geometric components at the same time, as in [14], we use the general extended form of the generalized vielbein

$$
E^{A}{ }_{M}=\left(\begin{array}{ccc}
e_{a}{ }^{i} & -e_{a}{ }^{k} C_{k i} & -e_{a}{ }^{k} A_{k \beta} \\
-e^{a}{ }_{k} \tilde{C}^{k i} & e^{a}{ }_{i}+e^{a}{ }_{k} \tilde{C}^{k j} C_{j i} & -e^{a}{ }_{k} \tilde{A}^{k}{ }_{\beta} \\
\tilde{A}^{i \alpha} & A_{i}{ }^{\alpha} & \delta^{\alpha}{ }_{\beta}
\end{array}\right)
$$

which combines (2.17) and (3.13) into one object. Recall that $\eta_{A B}=E_{A}{ }^{M} E_{M B}$ implies that the Weitzenböck connection satisfies $\Omega_{A B C}=-\Omega_{A C B}$. However, one can show that this relation ceases to be satisfied with the full vielbein (3.21). Therefore, in the following we present the geometric fluxes for the physically relevant case of $\tilde{A}^{i}{ }_{\alpha}=0$ and the nongeometric fluxes for $A_{i}{ }^{\alpha}=0$. In addition, for simplicity here we will work in a holonomic basis, the rather lengthy generalizations to a non-holonomic basis can be found in appendix B. The components of the derivatives $D_{A}=E_{A}{ }^{M} D_{M}$ in the holonomic basis become

$$
\begin{aligned}
\tilde{D}^{i} & =\tilde{\partial}^{i}+\tilde{C}^{i m} C_{m n} \tilde{\partial}^{n}-\tilde{C}^{i m} \partial_{m}-\tilde{A}^{i \gamma} \partial_{\gamma} \\
D_{i} & =\partial_{i}-C_{i m} \tilde{\partial}^{m}-A_{i}^{\gamma} \partial_{\gamma} \\
D_{\alpha} & =\partial_{\alpha}+A_{m \alpha} \tilde{\partial}^{m}+\tilde{A}^{m}{ }_{\alpha} \partial_{m} .
\end{aligned}
$$

For all three indices being of normal or winding type we get the fluxes $H, F, Q$ and $R$ including corrections depending on the gauge fields $A$ and $\tilde{A}$. In terms of the derivatives (3.22), for $\tilde{A}^{i}{ }_{\alpha}=0$ the geometric fluxes can be expressed as

$$
\begin{aligned}
& \mathcal{H}_{i j k}=-3 D_{\left[\underline{\underline{i}} B_{j k]}\right.}+3 D_{[\underline{[}} A_{\underline{j} \gamma} A_{\underline{k}]}{ }^{\gamma} \\
& F^{k}{ }_{i j}=-\tilde{D}^{k} B_{i j}+\tilde{D}^{k} A_{[\underline{i} \gamma} A_{\underline{j}]}^{\gamma}-2 D_{[\underline{[}} \beta^{k m} A_{\underline{j}] \gamma} A_{m}^{\gamma}-2 \beta^{k m} D_{[\underline{i}} C_{m \underline{j}]} .
\end{aligned}
$$


With $A_{i}{ }^{\alpha}=0$ the non-geometric fluxes take the form

$$
\begin{aligned}
& Q_{k}^{i j}=-D_{k} \beta^{i j}+D_{k} \tilde{A}^{[\underline{i} \gamma} \tilde{A}^{j]} \gamma-\tilde{C}^{[\underline{i m}} \tilde{C}^{j] n} D_{k} B_{m n}-2 D^{[\underline{i}} B_{k m} \tilde{C}^{j]} \underline{-}^{j] m} \\
& \left.R^{i j k}=-3 \tilde{D}^{[\underline{i}} \beta \underline{j k}\right]+3 \tilde{D}^{[\underline{i}} \tilde{A}^{\underline{j}} \tilde{A}^{\underline{k}]} \gamma+3 \tilde{C}^{[\underline{i} m} \tilde{D}^{\underline{j}} B_{m n} \tilde{C}^{\underline{k}] n}
\end{aligned}
$$

For $A_{i}{ }^{\alpha}=\tilde{A}^{i}{ }_{\alpha}=0$, these expressions are consistent with the ones derived in [14, 44, 45].

Due to the extra gauge coordinates $y^{\alpha}$ in heterotic DFT, there exist new types of fluxes. Choosing at least one index of $\mathcal{F}_{A B C}$ to be a gauge index, the antisymmetry $\Omega_{A B C}=$ $-\Omega_{A C B}$ in all indices forces us to set either $\beta^{i j}=\tilde{A}^{i}{ }_{\alpha}=0$ or $B_{i j}=A_{i}{ }^{\alpha}=0$. Of course, one can choose these constraints independently for each direction $(i j)$ or $(i)$, respectively. In the following, we present the result for choosing the same set of conditions for all directions.

Thus, in the geometric frame $\beta^{i j}=\tilde{A}_{\alpha}^{i}=0$, we get the following three types of non-vanishing gauge fluxes

$$
\begin{aligned}
G_{\alpha i j} & =-2 D_{[\underline{i}} A_{\underline{j}] \alpha}-D_{\alpha} B_{i j}+D_{\alpha} A_{[\underline{[}}{ }^{\gamma} A_{\underline{j}] \gamma} \\
J^{j}{ }_{\alpha i} & =\tilde{\partial}^{j} A_{i \alpha} \\
K_{\alpha \beta i} & =2 D_{[\underline{\alpha}} A_{i \underline{\beta}]} .
\end{aligned}
$$

Solving the strong constraint via $\tilde{\partial}^{i}=\partial_{\alpha}=0$, the first flux reduces to the familiar form of the field strength (2.2) for an abelian field. In the non-geometric frame $B_{i j}=A_{i}{ }^{\alpha}=0$, the non-vanishing fluxes are

$$
\begin{aligned}
J^{j}{ }_{\alpha i} & =-\partial_{i} \tilde{A}^{j}{ }_{\alpha} \\
\tilde{G}_{\alpha}{ }^{i j} & =-2 \tilde{D}^{\left[\underline{\underline{A}} \tilde{A}^{j}\right]}{ }_{\alpha}-D_{\alpha} \beta^{i j}+D_{\alpha} \tilde{A}^{[\underline{i} \gamma} \tilde{A}^{j]} \gamma \\
\tilde{K}^{\alpha \beta i} & =2 D^{[\underline{\alpha}} \tilde{A}^{i \underline{\beta}]} .
\end{aligned}
$$

Hence, the flux $J^{j}{ }_{\alpha i}$ in the non-geometric frame is indeed the flux we encountered in the previous section after applying one T-duality. Similarly, reducing $\tilde{G}_{\alpha}^{i j}$ for $\partial_{i}=\partial_{\alpha}=0$, one obtains

$$
\tilde{G}_{\alpha}{ }^{i j}=-2 \tilde{\partial}^{[\underline{i}} \tilde{A}^{j]}{ }_{\alpha},
$$

the gauge flux of $\tilde{A}$ found in the background after applying two T-dualities.

For a non-holonomic basis, one finds the commutators

$$
\begin{aligned}
{\left[\partial_{a}, \partial_{b}\right] } & =f_{a b}^{c} \partial_{c}, \quad \text { with } \quad f_{a b}^{c}:=e_{i}^{c}\left(\partial_{a} e_{b}{ }^{i}-\partial_{b} e_{a}^{i}\right) . \\
{\left[\tilde{\partial}^{a}, \tilde{\partial}^{b}\right] } & =\tilde{f}_{c}^{a b} \tilde{\partial}^{c}, \quad \text { with } \quad \tilde{f}_{c}^{a b}:=e_{c}^{i}\left(\tilde{\partial}^{a} e_{i}^{b}-\tilde{\partial}^{b} e_{i}{ }^{a}\right)
\end{aligned}
$$

providing correction terms to the fluxes shown above. The resulting rather lengthy expressions for these fluxes can be found in appendix B.

The upshot of the explicit analysis of this section is that, for the heterotic string, the T-dual of the constant gauge flux background on a flat geometry is a non-geometric background. Therefore, the concept of non-geometry does not only apply to closed string three-form backgrounds but also to gauge flux backgrounds. Moreover, we have seen that for the description of these T-dual backgrounds, it is appropriate to change to a nongeometric frame, where in particular the gauge 1-form $A=A_{i} d x^{i}$ is replaced by a gauge 1-vector $\tilde{A}=\tilde{A}^{i} \partial_{i}$. 


\subsection{Comment on $R$-flux and non-associativity}

It has been suggested that the non-geometric $R$-flux background gives rise to some nonassociativity of the usual coordinates [46-50]. In the context of DFT this was analyzed in [51], where it was studied how DFT behaves if one defines a new tri-product given in terms of the three-index flux as

$$
f \triangle g \triangle h=f g h+\mathcal{F}_{A B C} D^{A} f D^{B} g D^{C} h+\ldots .
$$

For objects satisfying the strong constraint, the correction identically vanishes so that, in particular, for the action such a deformation has no effect (see [51] for more details).

Since the coordinates themselves are not conformal fields, one does not necessarily expect them to satisfy the level-matching constraint and consequently not the strong constraint. The implied tri-bracket $\left[x^{i}, x^{j}, x^{k}\right]$ for the coordinates is governed by the nongeometric flux coupled to just ordinary derivatives. Thus, we are focusing on the term

$$
\mathcal{F}_{A B C} D^{A} f D^{B} g D^{C} h=\rho^{i j k} \partial_{i} f \partial_{j} g \partial_{k} h+\ldots
$$

which for usual DFT was just $\rho_{b o s}^{i j k}=3 \tilde{\partial}[\underline{\underline{i}} \beta \underline{\underline{j k}]}$. The natural expectation is that, in the heterotic case, this gets generalized to the gauge invariant combination

$$
\rho_{H}^{i j k}=3\left(\tilde{\partial}^{[\underline{i}} \beta \underline{\underline{j k}]}-\tilde{\partial}^{[\underline{\underline{i}}} \tilde{A}^{j \gamma} \tilde{A}^{\underline{k}} \gamma\right) .
$$

However, evaluating (3.30) for a holonomic non-geometric frame, one finds ${ }^{3}$

$$
\rho^{i j k}=3\left(\tilde{\partial}^{[\underline{i}} E_{A^{\underline{j}}}\right) E^{A \underline{k}]}=-3\left(\tilde{\partial}^{[\underline{i}} \beta^{\underline{j k}]}+\tilde{\partial}^{[\underline{i}} \tilde{A}^{\underline{j} \gamma} \tilde{A}^{\underline{k}} \gamma\right),
$$

showing that the relative sign between the two terms on the right hand side of (3.32) is different. As a consequence, this object $\rho^{i j k}$ is not invariant under $\tilde{A}$ gauge transformations $\tilde{A}^{i}{ }_{\alpha}=\tilde{A}^{i}{ }_{\alpha}+\tilde{\partial}^{i} \lambda_{\alpha}$, unless the non-geometric gauge flux $\tilde{G}_{\alpha}{ }^{i j}=\tilde{\partial}^{[\underline{i}} \tilde{A}_{-}^{j]}{ }_{\alpha}$ vanishes. This result is puzzling and deserves more study which is beyond the scope of this paper. Thus we leave it as an observation.

\subsection{Comment on S-duality}

Let us now consider the $\mathrm{SO}(32)$ heterotic string compactified on a two-torus with constant abelian gauge flux $F=F_{12}$. This configuration is known to be S-dual to the Type I string [52] compactified on a two-torus where the $D 9$-brane carries the same gauge flux $F$. Applying a T-duality in the $y$-direction to this latter configuration yields the Type I' string with a $D 8$-brane at an angle with respect to the O8-planes. One might ask whether there exist an S-dual to this configuration.

The answer to this question is not obvious, as in the heterotic string there are no 8-branes. Usually, under a T-duality the $\mathrm{SO}(32)$ heterotic string maps to the $E_{8} \times E_{8}$ heterotic string. Moreover, we have just seen that the T-dual of an heterotic string with gauge flux is a non-geometric background carrying flux $J=J^{1}{ }_{2}$. Therefore, by completing the diagram as shown in figure 1 we are led to the conjecture that the S-dual of the D8brane at angle in Type I' is a non-geometric $J$-flux background of the heterotic string.

\footnotetext{
${ }^{3}$ We confirmed this behavior by performing a conformal field theory analysis along the lines of [49].
} 


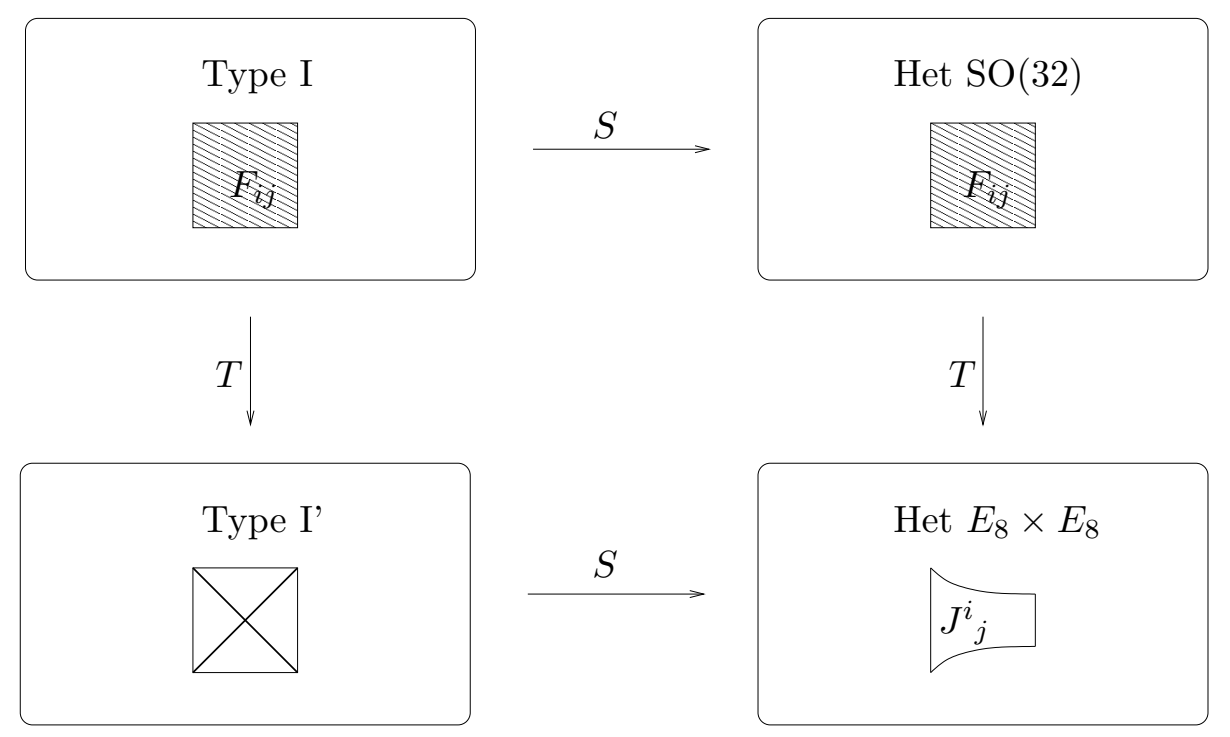

Figure 1. $S$ - and $T$-duality between Type I and heterotic string.

\section{A Lie algebroid for heterotic field redefinitions}

In the previous section we have seen that a field redefinition can help in simplifying the description of non-geometric backgrounds. As we discussed in the introduction, this fact is familiar from $O(D, D)$ generalized geometry and DFT, respectively. Recall that in [25] the general structure of $O(D, D)$ induced field definitions was clarified in the framework of generalized geometry. The two main results were that for every such field redefinition, one can associate a corresponding Lie algebroid so that the redefined supergravity action is governed by the differential geometry of that Lie algebroid.

In this section, we show that this picture also holds for the heterotic case, i.e. to every $O(D, D+n)$ induced field redefinition one can associate a corresponding Lie algebroid so that in the new field variables the heterotic action is governed by the differential geometry of that Lie algebroid. For the definition of a Lie algebroid, please consult appendix C. We will also show that the non-geometric frame (3.12) does also fit into this scheme. Since the story is very similar, we will be rather brief here and refer the reader to [25] for more information on Lie algebroids and its differential geometry.

\section{1 $O(D, D+n)$-induced field redefinition}

In abelian heterotic generalized geometry, one considers a D-dimensional manifold $M$ with usual coordinates $x^{i}$, equipped with a generalized bundle $E=T M \oplus T^{*} M \oplus V$, whose sections are formal sums $\xi+\tilde{\xi}+\lambda$ of vectors, $\xi=\xi^{i}(x) \partial_{i}$, one-forms, $\tilde{\xi}=\tilde{\xi}_{i}(x) d x^{i}$ and gauge transformations, $\lambda=\left(\lambda_{1}(x), \ldots \lambda_{n}(x)\right)$, of $\mathrm{U}(1)^{n}$. On this bundle one defines a generalized $\mathcal{H}_{M N}$ metric taking the familiar form (2.12) in terms of the fundamental fields $g_{i j}, B_{i j}$ and $A_{i}{ }^{\alpha}$. An $O(D, D+n)$ transformation $\mathcal{M}$ acts on the generalized metric via conjugation, i.e.

$$
\hat{H}(\hat{g}, \hat{B}, \hat{A})=\mathcal{M}^{t} H(g, B, A) \mathcal{M}
$$


and therefore defines a field redefinition

$$
(g, B, A) \longrightarrow(\hat{g}, \hat{B}, \hat{A}) .
$$

The heterotic action in terms of the fields $(g, B, A)$ is the heterotic supergravity action (2.1). The question is how the action in the new field variables $(\hat{g}, \hat{B}, \hat{A})$ looks. Just inserting the field redefinition gives a plethora of terms so that an organizing principle is needed.

To proceed, we write a general $O(D, D+n)$ matrix $\mathcal{M}$ as

$$
\mathcal{M}=\left(\begin{array}{lll}
a & b & m \\
c & d & n \\
p & q & z
\end{array}\right) .
$$

This transformation has to leave the $\eta$ metric (2.6) invariant, i.e.

$$
\mathcal{M}^{t} \eta \mathcal{M}=\eta,
$$

leading to six independent constraints on the submatrices

$$
\begin{aligned}
c^{t} a+a^{t} c+p^{t} p & =0 \\
c^{t} b+a^{t} d+p^{t} q & =1 \\
c^{t} m+a^{t} n+p^{t} z & =0 \\
d^{t} b+b^{t} d+q^{t} q & =0 \\
d^{t} m+b^{t} n+q^{t} z & =0 \\
n^{t} m+m^{t} n+z^{t} z & =1 .
\end{aligned}
$$

Now applying (4.1) we can read off the induced field redefinition. For the upper-left component of $\hat{H}(\hat{g}, \hat{B}, \hat{A})$ one obtains

$$
\hat{H}(\hat{g}, \hat{B}, \hat{A})_{u l}=\left[a-A p-\left(g+B+\frac{1}{2} A^{2}\right) c\right]^{t} g^{-1}\left[a-A p-\left(g+B+\frac{1}{2} A^{2}\right) c\right]
$$

which, comparing with general form of the generalized metric, gives $\hat{g}^{-1}$. Thus, we get

$$
\hat{g}=\left(\gamma^{-1}\right) g\left(\gamma^{-1}\right)^{t}
$$

where the matrix $\gamma$ is given as

$$
\gamma=a-A p-\left(g+B+\frac{1}{2} A^{2}\right) c .
$$

In order to consider the redefined Kalb-Ramond field $\hat{B}$ which is contained in $\hat{C}$, we consider the upper-middle component of the redefined generalized metric

$$
\hat{H}(\hat{g}, \hat{B}, \hat{A})_{u m}=1+\left[a-A p-\left(g+B+\frac{1}{2} A^{2}\right) c\right]^{t} g^{-1}\left[b-A q-\left(g+B+\frac{1}{2} A^{2}\right) d\right]
$$


and compare it with $\hat{H}_{u m}=-\hat{g}^{-1} \hat{C}$. Thus, we find

$$
\hat{C}=\left(\gamma^{-1}\right) \mathfrak{C}\left(\gamma^{-1}\right)^{t} \quad \text { with } \quad \mathfrak{C}=\delta \gamma^{t}-g
$$

with the matrix $\delta$ defined as

$$
\delta=-b+A q+\left(g+B+\frac{1}{2} A^{2}\right) d .
$$

It remains to determine the $O(D, D+n)$ induced field redefinition for the gauge field $A$. For that purpose, we look into the upper-right element of the generalized metric

$$
\hat{H}(\hat{g}, \hat{B}, \hat{A})_{u r}=\left[a-A p-\left(g+B+\frac{1}{2} A^{2}\right) c\right]^{t} g^{-1}\left[m-A z-\left(g+B+\frac{1}{2} A^{2}\right) n\right]
$$

and identify it with $-\hat{g}^{-1} \hat{A}$. Thus, we obtain

$$
\hat{A}=\left(\gamma^{-1}\right) \mathfrak{A}
$$

with

$$
\mathfrak{A}=-m+A z+\left(g+B+\frac{1}{2} A^{2}\right) n .
$$

From $\mathfrak{C}$ and $\mathfrak{A}$ one can define also a new $B$-field $\mathfrak{B}$ via

$$
\hat{B}=\left(\gamma^{-1}\right) \mathfrak{B}\left(\gamma^{-1}\right)^{t} \quad \text { with } \quad \mathfrak{B}=\mathfrak{C}-\frac{1}{2} \mathfrak{A} \otimes \mathfrak{A} .
$$

Thus the field redefinition is of a very peculiar form, where the matrix $\gamma$ plays a prominent role. In fact, the structure of the field redefinition of $g$ and $B$ is completely analogous to [25], only containing some new gauge field dependent corrections in $\gamma$ and $\delta$. Thus it is straightforward to proceed as in [25] and to identify

$$
\rho=\left(\gamma^{-1}\right)^{t}
$$

as the anchor map of a Lie algebroid (see appendix C).

This Lie algebroid lives on the tangent bundle itself, i.e. $E=T M$ and the anchor map $\rho: E \rightarrow T M$ acts on a vector field $X=X^{i} \partial_{i} \in E$ as $^{4}$

$$
\rho(X)=\left(\rho_{j}^{i} X^{j}\right) \partial_{i}=X^{i}\left(\rho^{t}\right)_{i}{ }^{j} \partial_{j}=X^{i} D_{i}
$$

where we defined the partial derivative for the Lie algebroid as

$$
D_{i}=\left(\rho^{t}\right)_{i}^{j} \partial_{j}
$$

The bracket $\llbracket \cdot, \cdot \rrbracket$ on $E=T M$ is defined as

$$
\llbracket X, Y \rrbracket=\left(X^{j} D_{j} Y^{k}-Y^{j} D_{j} X^{k}+X^{i} Y^{j} F_{i j}^{k}\right) \partial_{k} .
$$

\footnotetext{
${ }^{4}$ Here we present the relations in a holonomic basis. For the non-holonomic case, we refer to [25].
} 
with the structure constants

$$
F_{i j}{ }^{k}=\left(\rho^{-1}\right)^{k}{ }_{m}\left(D_{i}\left(\rho^{t}\right)_{j}^{m}-D_{j}\left(\rho^{t}\right)_{i}^{m}\right) .
$$

Indeed, this bracket satisfies the homomorphism property

$$
\rho(\llbracket X, Y \rrbracket)=[\rho(X), \rho(Y)]
$$

Furthermore, by construction the new bracket $\llbracket \cdot, \cdot \rrbracket$ satisfies the Jacobi identity (C.2) as well as the Leibniz rule (C.1). Thus, for every $O(D, D+n)$ induced field redefinition we have associated a corresponding Lie algebroid. The true power of this formal approach will become clear in the next section.

\subsection{The redefined heterotic action}

Recall that the NS-sector of the heterotic DFT action is

$$
\mathcal{S}=\int d x \sqrt{g} e^{-2 \phi}\left(R+4(\partial \phi)^{2}-\frac{1}{12} H^{i j k} H_{i j k}-\frac{1}{4} G^{i j \alpha} G_{i j \alpha}\right)
$$

with the three-form $H=d B-\frac{1}{2} \delta_{\alpha \beta} A^{\alpha} \wedge d A^{\beta}$ and the abelian two-form field strength $G^{\alpha}=d A^{\alpha}$. As derived in detail in [25], the field redefinition is completely given by pulling indices up and down by the action of the anchor (here $\rho^{t}=\gamma^{-1}$ ). For the metric we found (4.7), which implies that the quantities in the gravitational sector transform as

$$
\begin{array}{rlrl}
\hat{R}_{m n p}^{q} & =\left(\rho^{-1}\right)^{q} l \rho_{m}^{i} \rho_{n}^{j} \rho_{p}^{k} R_{i j k}^{l}, \quad \hat{R}_{m n}=\rho_{m}^{i} \rho_{n}^{j} R_{i j}, \\
\hat{R} & =R, \quad \sqrt{|\hat{g}|}=\sqrt{|g|} \rho^{t} \mid, \quad \hat{\phi} & =\phi
\end{array}
$$

where the derivative for the transformed theory is (4.18).

For the flux sector, so far we know the transformation behavior of gauge potentials $B$ and $A$. Therefore, one still needs to find the proper definition of the new field strengths so that they also transform properly, i.e. just by pulling up and down indices with the anchor. For that purpose one needs to invoke the Lie algebroid differential $d_{E}$ defined in appendix C. For the gauge field strength $G=d A$, using the relation (C.4) one can show

$$
\left(\Lambda^{2} \rho^{*}\right) d_{E} \hat{\mathfrak{A}}=d\left(\rho^{*} \hat{\mathfrak{A}}\right)=d A
$$

with $\rho^{*}=\left(\rho^{t}\right)^{-1}=\gamma$, so that

$$
\hat{\mathfrak{G}}:=d_{E} \hat{\mathfrak{A}}=\left(\Lambda^{2} \rho^{t}\right) G
$$

is the correct definition of the transformed field strength that transforms properly. Analogously, one can show

$$
d_{E} \hat{\mathfrak{B}}=\left(\Lambda^{3} \rho^{t}\right) d B
$$

so that the proper three-form flux is given by

$$
\hat{\mathfrak{H}}:=d_{E} \hat{\mathfrak{B}}-\frac{1}{2} \hat{\mathfrak{A}} \wedge d_{E} \hat{\mathfrak{A}}=\left(\Lambda^{3} \rho^{t}\right) H .
$$


Its Bianchi identity reads

$$
d_{E} \hat{\mathfrak{H}}=-\frac{1}{2} \hat{\mathfrak{G}} \wedge \hat{\mathfrak{G}}
$$

Thus, each quantity appearing in the heterotic action (4.22) now transforms properly so that the action in the redefined fields can be expressed as

$$
\mathcal{S}=\int d x \sqrt{\hat{g}}\left|\rho^{*}\right| e^{-2 \phi}\left(\hat{R}+4(D \phi)^{2}-\frac{1}{12} \hat{\mathfrak{H}}^{i j k} \hat{\mathfrak{H}}_{i j k}-\frac{1}{4} \hat{\mathfrak{G}}^{i j \alpha} \hat{\mathfrak{G}}_{i j \alpha}\right)
$$

This has the analogous form as the original action, but with the new fields defined in the framework of the differential geometry of the Lie algebroid. Therefore, the latter provides the organizing principle for expressing the action in $O(D, D+n)$ induced redefined field variables.

Note that the symmetries of this action are just the transformed diffeomorphisms and $B$ - and $A$-field gauge transformations of the original action. Clearly, just by a field redefinition, one does not gain new symmetries. Therefore, the $\tilde{A}$ field gauge transformation (3.16), needed for the transition function of the T-dual non-geometric $J$-flux background is not a symmetry of (4.29). Thus, as in generalized geometry [25], a field redefinition helps to bring in each patch a non-geometric background in a simple form, but in general it does not provide a global description of the background.

\subsection{The non-geometric frame}

In this section we show that the field redefinition between the geometric and the nongeometric frame from section 3.1 can also be described in this framework. For that purpose, first recall the form of the generalized metric in these two frames. In the geometric one, we have

$$
\mathcal{H}_{M N}=\left(\begin{array}{ccc}
g^{i j} & -g^{i k} C_{k j} & -g^{i k} A_{k \beta} \\
-g^{j k} C_{k i} & g_{i j}+C_{k i} g^{k l} C_{l j}+A_{i}^{\gamma} A_{j \gamma} & C_{k i} g^{k l} A_{l \beta}+A_{i \beta} \\
-g^{j k} A_{k \alpha} & C_{k j} g^{k l} A_{l \alpha}+A_{j \alpha} & \delta_{\alpha \beta}+A_{k \alpha} g^{k l} A_{l \beta}
\end{array}\right)
$$

and in the non-geometric one

$$
\mathcal{H}_{M N}=\left(\begin{array}{ccc}
\tilde{g}^{i j}+\tilde{C}^{k i} \tilde{g}_{k l} \tilde{C}^{l j}+\tilde{A}^{i}{ }_{\gamma} \tilde{A}^{j \gamma}-\tilde{g}_{j k} \tilde{C}^{k i} & \tilde{C}^{k i} \tilde{g}_{k l} \tilde{A}_{\beta}^{l}+\tilde{A}^{i}{ }_{\beta} \\
-\tilde{g}_{i k} \tilde{C}^{k j} & \tilde{g}_{i j} & -\tilde{g}_{i k} \tilde{A}^{k}{ }_{\beta} \\
\tilde{C}^{k j} \tilde{g}_{k l} \tilde{A}_{\alpha}^{l}+\tilde{A}^{j}{ }_{\alpha} & -\tilde{g}_{j k} \tilde{A}_{\alpha}{ }_{\alpha} \delta_{\alpha \beta}+\tilde{A}^{k}{ }_{\alpha} \tilde{g}_{k l} \tilde{A}_{\beta}^{l}{ }
\end{array}\right) .
$$

By comparison of the components, the corresponding field redefinition takes the form

$$
\begin{aligned}
\tilde{g} & =g+C^{t} g^{-1} C+A^{2} \\
\tilde{C} & =\tilde{g}^{-1} C^{t} g^{-1} \\
\tilde{A} & =-\left(\tilde{g}^{-1}+\tilde{C}\right) A .
\end{aligned}
$$

Analogous to [25], we propose that this transformation is implemented by choosing

$$
\mathcal{M}=\left(\begin{array}{ccc}
0 & \tilde{g} & 0 \\
\tilde{g}^{-1} & 0 & 0 \\
0 & 0 & 1
\end{array}\right)
$$


with $\tilde{g}=g+C^{t} g^{-1} C+A^{2}$. Evaluating the expressions (4.8), (4.10), (4.11), (4.14) we obtain as intermediate results

$$
\begin{aligned}
& \gamma=-(g+C) \tilde{g}^{-1} \quad \text { so that } \quad \gamma^{-1}=-\left(g+C^{t}\right) g^{-1}, \\
& \delta=-\tilde{g} \quad \text { so that } \quad \mathfrak{C}=C^{t} \text {, } \\
& \mathfrak{A}=A \text {. }
\end{aligned}
$$

Using these relations further in (4.7), (4.10) and (4.13) we finally get

$$
\begin{aligned}
\hat{g} & =\left(\gamma^{-1}\right) g\left(\gamma^{-1}\right)^{t}=\tilde{g} \\
\hat{C} & =\left(\gamma^{-1}\right) \mathfrak{C}\left(\gamma^{-1}\right)^{t}=C^{t} g^{-1} \tilde{g} \\
\hat{A} & =\left(\gamma^{-1}\right) \mathfrak{A}=-\left(1+C^{t} g^{-1}\right) A .
\end{aligned}
$$

Here $\hat{C}$ and $\hat{A}$ are still forms. For transforming them into a bi-vector and a vector, one pulls up the indices with $\tilde{g}^{-1}$ so that

$$
\begin{aligned}
\tilde{C} & =\tilde{g}^{-1} \hat{C} \tilde{g}^{-1}=\tilde{g}^{-1} C^{t} g^{-1} \\
\tilde{A} & =\tilde{g}^{-1} \hat{A}=-\left(\tilde{g}^{-1}+\tilde{C}\right) A,
\end{aligned}
$$

which precisely agrees with the field redefinition of the non-geometric frame (4.32).

\section{Conclusion}

In this paper we studied a couple of aspects of heterotic DFT in more detail. We think that, while the general formalism of heterotic DFT was developed before and is a straightforward generalization of bosonic DFT, the concrete evaluation of its consequences, in particular for issues related to the gauge field, deserved a further study.

Indeed, by applying the T-duality rules ( $\alpha^{\prime}$ corrected heterotic Buscher rules) to a flat background with a constant gauge field, we found non-geometric backgrounds, which were very similar to the $Q$ - and $R$-flux backgrounds in bosonic DFT. Namely, after one T-duality we already obtained a background which was best described by changing to a non-geometric frame, where the gauge one-form has turned into a gauge one-vector. The required transition function between two patches was given by a new symmetry, namely a one-vector gauge transformation involving a winding dependence. Thus, this background is globally non-geometric, an effect introduced by the $\alpha^{\prime}$ corrected Buscher rules. Applying a further T-duality, the arising background was even locally non-geometric.

Even though, we were only considering abelian gauge fields, we expect this picture to generalize also to non-abelian gauge fields. The latter are introduced via a gauging procedure that generically breaks the $O(D, D+n)$ symmetry to $O(D, D)$. However, T-duality is a special element of $O(D, D)$ so that it can still be treated analogously to the abelian case.

Moreover, we clarified which type of fluxes are turned on in these backgrounds and how they are microscopically described in terms of the fundamental fields in the theory. We argued that the constant non-geometric $J$-flux background of the $E_{8} \times E_{8}$ heterotic string can be considered the S-dual of a Type I' background with a D8-brane intersecting the $O 8$-plane at an angle. 
Led by the apparent necessity of field redefinitions, we considered the general question what effect an $O(D, D+n)$ induced field redefinition has on the heterotic supergravity action. Generalizing [25], we investigated this question in the framework of generalized geometry and found very similar results, though now including various corrections due to the present one-form gauge field. In particular, the organizing principle for the terms in the redefined action was given by the differential geometry of a Lie algebroid, whose anchor was related to the $O(D, D+n)$ transformation. The non-geometric frame was identified with just a specific $O(D, D+n)$ induced field redefinition.

Even though, here we were only considering the NS part of the heterotic action, we expect that the whole action including the fermionic terms are governed by the objects in the differential geometry of the Lie algebroid. This includes e.g. the kinetic terms for the gravitinos and gluinos, that involve a spin-connection. Moreover, here we were neglecting the gravitational Chern-Simons term (see [35] for a recent treatment in DFT). Introducing non-abelian gauge fields via gauging, breaks the $O(D, D+n)$ symmetry so that in this case only the remaining symmetry should be used for a field redefinition.

\section{Acknowledgments}

We would like to thank Andre Betz, Michael Fuchs, Daniela Herschmann, Dieter Lüst, Felix Rennecke and Christian Schmid for discussions. R.B. would like to express a special thanks to the Mainz Institute for Theoretical Physics (MITP) for its hospitality and support. R.S. would also like to thank Klaus Altmann for support. The work of R.S. is supported by the China Scholarship Council (CSC).

\section{A The Buscher rules derived from heterotic DFT}

Using the implementation of T-duality in heterotic DFT, one can now quite generally (re)derive the Buscher from the conjugation of the generalized metric with the corresponding T-duality matrix. Carrying out this procedure for a T-duality in the $x^{\theta}$ direction, we get precisely the $\alpha^{\prime}$ corrected Buscher rules presented in [42]

$$
\begin{aligned}
G_{\theta \theta}^{\prime}= & \frac{G_{\theta \theta}}{\left(G_{\theta \theta}+\frac{\alpha^{\prime}}{2} A_{\theta}^{2}\right)^{2}} \\
G_{\theta i}^{\prime}= & -\frac{G_{\theta \theta} B_{\theta i}+\frac{\alpha^{\prime}}{2} G_{\theta i} A_{\theta}^{2}-\frac{\alpha^{\prime}}{2} G_{\theta \theta} A_{\theta} A_{i}}{\left(G_{\theta \theta}+\frac{\alpha^{\prime}}{2} A_{\theta}^{2}\right)^{2}} \\
G_{i j}^{\prime}= & G_{i j}-\frac{G_{\theta i} G_{\theta j}-B_{\theta i} B_{\theta j}}{\left(G_{\theta \theta}+\frac{\alpha^{\prime}}{2} A_{\theta}^{2}\right)} \\
& -\frac{1}{\left(G_{\theta \theta}+\frac{\alpha^{\prime}}{2} A_{\theta}^{2}\right)^{2}}\left(G_{\theta \theta}\left[\frac{\alpha^{\prime}}{2} B_{\theta j} A_{\theta} A_{i}+\frac{\alpha^{\prime}}{2} B_{\theta i} A_{\theta} A_{j}-\frac{\alpha^{\prime 2}}{4} A_{\theta} A_{i} A_{\theta} A_{j}\right]\right. \\
& \left.\quad+\frac{\alpha^{\prime}}{2} A_{\theta}^{2}\left[\left(G_{\theta i}-B_{\theta i}\right)\left(G_{\theta j}-B_{\theta j}\right)+\frac{\alpha^{\prime}}{2}\left(G_{\theta i} A_{\theta} A_{j}+G_{\theta j} A_{\theta} A_{i}\right)\right]\right)
\end{aligned}
$$




$$
\begin{aligned}
B_{\theta i}^{\prime} & =-\frac{G_{\theta i}+\frac{\alpha^{\prime}}{2} A_{\theta} A_{i}}{\left(G_{\theta \theta}+\frac{\alpha^{\prime}}{2} A_{\theta}^{2}\right)} \\
B_{i j}^{\prime} & =B_{i j}-\frac{\left(G_{\theta i}+\frac{\alpha^{\prime}}{2} A_{\theta} A_{i}\right) B_{\theta j}-\left(G_{\theta j}+\frac{\alpha^{\prime}}{2} A_{\theta} A_{j}\right) B_{\theta i}}{\left(G_{\theta \theta}+\frac{\alpha^{\prime}}{2} A_{\theta}^{2}\right)} \\
A^{\prime}{ }^{\alpha} & =-\frac{A_{\theta}{ }^{\alpha}}{\left(G_{\theta \theta}+\frac{\alpha^{\prime}}{2} A_{\theta}^{2}\right)} \\
A^{\prime}{ }^{\alpha}{ }^{\alpha} & =A_{i}{ }^{\alpha}-A_{\theta}{ }^{\alpha} \frac{G_{\theta i}-B_{\theta i}+\frac{\alpha^{\prime}}{2} A_{\theta} A_{i}}{\left(G_{\theta \theta}+\frac{\alpha^{\prime}}{2} A_{\theta}^{2}\right)}
\end{aligned}
$$

where e.g. $A_{\theta} A_{i}=A_{\theta}^{\alpha} A_{i \alpha}$. Here the metric and the Kalb-Ramond field have dimension $[l]^{0}$ and the gauge field $[l]^{-1}$.

\section{B Non-holonomic fluxes for heterotic DFT}

In this appendix we present the explicit expressions of the fluxes in a a non-holonomic basis. From the generalized vielbein $E_{A}^{M}$ and the dilation $d$ one can build the generalized fluxes

$$
\begin{aligned}
\mathcal{F}_{A B C} & =E_{C M} \mathcal{L}_{E_{A}} E_{B}{ }^{M}=\Omega_{A B C}+\Omega_{C A B}-\Omega_{B A C} \\
\mathcal{F}_{A} & =-e^{2 d} \mathcal{L}_{E_{A}} e^{-2 d}=-\partial_{M} E_{A}{ }^{M}+2 D_{A} d .
\end{aligned}
$$

The generalized derivative $D_{A}=E_{A}^{M} D_{M}$ takes the form

$$
\begin{aligned}
\tilde{D}^{a} & =\tilde{\partial}^{a}+\tilde{C}^{a m} C_{m n} \tilde{\partial}^{n}-\tilde{C}^{a m} \partial_{m}-\tilde{A}^{a \gamma} \partial_{\gamma} \\
D_{a} & =\partial_{a}-C_{a m} \tilde{\partial}^{m}-A_{a}{ }^{\gamma} \partial_{\gamma} \\
D_{\alpha} & =\partial_{\alpha}+A_{m \alpha} \tilde{\partial}^{m}+\tilde{A}^{m}{ }_{\alpha} \partial_{m} .
\end{aligned}
$$

As in section 3, we present the geometric fluxes for the physically relevant case of $\tilde{A}^{a}{ }_{\alpha}=0$ and the non-geometric fluxes for $A_{a}{ }^{\alpha}=0$. From the flux definition (B.1) we obtain the geometric fluxes ${ }^{5}$

$$
\begin{aligned}
& H_{a b c}=-3\left(D_{[\underline{a}} B_{\underline{b c}]}-D_{[\underline{a}} A_{\underline{b} \gamma} A_{\underline{c}]}^{\gamma}+f^{m}{ }_{[\underline{a b}} C_{\underline{c}] m}-C_{[\underline{a} m} C_{\underline{b} n} \tilde{f}_{\underline{c}]} m n-A_{[\underline{a}}^{\beta} \partial_{\beta} e_{\underline{b}}^{i} C_{\underline{c}] i}\right) \\
& F_{a b}^{c}=f_{a b}^{c}-\tilde{D}^{c} B_{a b}+\tilde{D}^{c} A_{[\underline{a} \gamma} A_{\underline{b}]}^{\gamma}+2 C_{[\underline{a} m} \tilde{f}_{\underline{b}]}{ }^{m c}-2 D_{[\underline{a}} \beta^{c m} A_{\underline{b}] \gamma} A_{m}^{\gamma}-2 \beta^{c m} D_{[\underline{a}} C_{m \underline{b}]} \\
& +3 \beta^{c m}\left(f_{[\underline{m a}}^{n} C_{\underline{b}] n}-C_{[\underline{m} n} C_{\underline{a} p} \tilde{f}_{\underline{b}]}^{n p}\right)-2 \beta^{c m}\left(C_{m i} A_{[\underline{a}}^{\beta} \partial_{\beta} e_{\underline{b}]}^{i}+C_{[\underline{a} i} A_{\underline{b}]}^{\beta} \partial_{\beta} e_{m}^{i}\right) \\
& -2 A_{[\underline{a}}^{\beta} \partial_{\beta} e_{\underline{b}}{ }^{i} e^{c}{ }_{i}
\end{aligned}
$$

\footnotetext{
${ }^{5}$ Note that the derivative $D^{i}, D_{i}$ and $D_{\alpha}$ will also be simplified.
} 
and for $A_{a}{ }^{\alpha}=0$ the non-geometric fluxes read

$$
\begin{aligned}
& Q_{c}{ }^{a b}=-D_{c} \beta^{a b}+D_{c} \tilde{A}^{[\underline{a} \gamma} \tilde{A}^{\underline{b}]} \gamma-2 \tilde{D}^{[\underline{a}} B_{c n} \tilde{C}^{\underline{b}] n}-\tilde{C}^{[\underline{a m}} \tilde{C}^{b] n} D_{c} B_{m n}+\tilde{f}_{c}^{a b}+2 \tilde{C}^{[\underline{a} m} f^{\underline{b}]} m c \\
& +2 B_{c m} \tilde{C}^{[\underline{a} n} \tilde{f}_{n}^{\underline{b}] m}+2 \tilde{C}^{[\underline{a} m} B_{m n} \tilde{f}_{c}^{n \underline{b}]}-3 \tilde{C}^{a m} \tilde{C}^{b n}\left(B_{[m p} f^{p}{ }_{n c]}-B_{[\underline{m p}} C_{\underline{n} q} \tilde{f}_{c]}^{p q}\right) \\
& +2\left(B_{c m} \tilde{C}^{[\underline{a} n} \tilde{A}^{\underline{b}] \gamma} \partial_{\gamma} e_{n}{ }^{i} e^{m}{ }_{i}+\tilde{A}^{[\underline{a} \gamma} \partial_{\gamma} e_{c}^{i} e^{\underline{b}]}{ }_{i}-\tilde{C}^{[\underline{a} m} B_{m i} \tilde{A}^{\underline{b}] \gamma} \partial_{\gamma} e_{c}^{i}\right) \\
& \left.R^{a b c}=-3 \tilde{D}^{[\underline{a}} \beta \underline{b c]}+3 \tilde{D}^{[\underline{a}} \tilde{A}^{\underline{b} \gamma} \tilde{A}^{c]}{ }_{\gamma}+3 \tilde{C}^{[\underline{a} m} \tilde{D}^{\underline{b}} B_{m n} \tilde{C}^{c] n}+6 \tilde{C}^{[\underline{a} m} \tilde{C}^{\underline{b} n} B_{[\underline{m p}} \tilde{f}_{\underline{n}]}^{p} \underline{\underline{C}}\right] \\
& +3 \tilde{C}^{a m} \tilde{C}^{b n} \tilde{C}^{c p}\left(B_{[\underline{m} q} f_{\underline{n p}]}^{q}-B_{[\underline{m} q} B_{\underline{n} l} \tilde{f}_{\underline{p}]}^{q l}\right)+3\left(\tilde{C}^{[\underline{a m}} \tilde{C}^{\underline{b n}} f^{c]}{ }_{m n}-\tilde{C}^{[\underline{a} m} f_{m}{ }^{\underline{b c}]}\right) \\
& +3\left(\tilde{C}^{[\underline{a} m} \tilde{C}^{\underline{\underline{b}} n} B_{n i} \tilde{A}^{\underline{c}] \gamma} \partial_{\gamma} e_{m}^{i}-2 \tilde{C}^{[\underline{\underline{a} m}} \tilde{A}^{\underline{\underline{b}} \gamma} \partial_{\gamma} e_{m}{ }^{i} e^{\underline{c}]}{ }_{i}\right) \text {. }
\end{aligned}
$$

For $A_{i}{ }^{\alpha}=\tilde{A}^{i}{ }_{\alpha}=0$, these expressions coincide with the ones derived in [14] and [45]. Similarly, the fluxes $\mathcal{F}_{A}$ can be expanded as

$$
\begin{aligned}
& F_{a}=-\partial_{m} e_{a}{ }^{m}+\tilde{\partial}^{m} C_{a m}+\partial_{\alpha} A_{a}^{\alpha}+2 D_{a} d \\
& F^{a}=\partial_{m} \tilde{C}^{a m}-\tilde{\partial}^{m} e^{a}{ }_{m}-\tilde{\partial}^{m}\left(\tilde{C}^{a n} C_{n m}\right)+\partial_{\alpha} \tilde{A}^{a \alpha}+2 \tilde{D}^{a} d .
\end{aligned}
$$

Due to the extra gauge coordinates in heterotic DFT, we also have the gauge fluxes $G_{\alpha a b}, J^{c}{ }_{\alpha b}$ and $\tilde{G}_{\alpha}{ }^{a b}$. For $\tilde{A}_{\alpha}^{a}=\beta=0$ they become

$$
\begin{aligned}
G_{\alpha a b}= & -D_{\alpha} B_{a b}+D_{\alpha} A_{[\underline{a}}^{\gamma} A_{\underline{b}] \gamma}-2 D_{[\underline{a}} A_{b] \alpha}+A_{\alpha m} f_{a b}^{m}+2 C_{[\underline{a} m} A_{n \alpha} \tilde{f}_{\underline{b}]} m n \\
& +2\left(C_{[\underline{a} i} \partial_{\alpha} e_{\underline{b}]}{ }^{i}-A_{\alpha i} A_{[\underline{\underline{a}}}^{\gamma} \partial_{\gamma} e_{\underline{b}]}^{i}\right) \\
J^{c}{ }_{\alpha b}= & \tilde{\partial}^{c} A_{b \alpha}+A_{m \alpha} \tilde{f}_{b}{ }^{c m}+\partial_{\alpha} e_{b}{ }^{i} e^{c}{ }_{i} \\
K_{\alpha \beta a}= & 2 D_{[\underline{\alpha}} A_{a \underline{\beta}]}+A_{m \alpha} A_{n \beta} \tilde{f}_{a}^{m n}+2 A_{i[\underline{\alpha}} \partial_{\underline{\beta}]} e_{a}^{i},
\end{aligned}
$$

while for $A_{a}{ }^{\alpha}=B=0$ they can be expanded as

$$
\begin{aligned}
& J_{\alpha b}^{c}=-\partial_{b} \tilde{A}_{\alpha}^{c}+\tilde{A}_{\alpha}^{m}{ }_{\alpha} f^{c}{ }_{m b}+\partial_{\alpha} e_{b}{ }^{i} e^{c}{ }_{i} \\
& \tilde{G}_{\alpha}{ }^{a b}=-D_{\alpha} \beta^{a b}+D_{\alpha} \tilde{A}^{[\underline{a} \gamma} \tilde{A}^{\underline{b}]}{ }_{\gamma}-2 \tilde{D}^{[\underline{a}} \tilde{A}_{\alpha}^{\underline{b}]}+\tilde{A}_{\alpha}^{m}{ }_{\alpha} \tilde{f}_{m}{ }^{a b}+2 \tilde{C}^{[\underline{a} m} \tilde{A}_{\alpha}^{n} f^{\underline{b}]}{ }_{m n} \\
& +2\left(\tilde{C}^{[\underline{a} i} \partial_{\alpha} e^{\underline{b}]}{ }_{i}-\tilde{A}_{\alpha}^{i} \tilde{A}^{[\underline{a} \gamma} \partial_{\gamma} e^{\underline{b}]}{ }_{i}\right) \\
& \left.\tilde{K}^{\alpha \beta a}=2 D^{[\underline{\alpha}} \tilde{A}^{a} \underline{\beta}\right]+\tilde{A}^{m \alpha} \tilde{A}^{n \beta} f^{a}{ }_{m n}+2 \tilde{A}^{i}\left[\underline{\alpha} \partial^{\underline{\beta}]} e^{a}{ }_{i} .\right.
\end{aligned}
$$

In addition, there exists the flux

$$
F_{\alpha}=-\partial_{m} \tilde{A}_{\alpha}^{m}-\tilde{\partial}^{m} A_{m \alpha}+2 D_{\alpha} d
$$

\section{Lie algebroids}

A Lie algebroid is specified by three pieces of information:

- a vector bundle $E$ over a manifold $M$,

- a bracket $[\cdot, \cdot]_{E}: E \times E \rightarrow E$, and

- a homomorphism $\rho: E \rightarrow T M$ called the anchor. 
Similar to the usual Lie bracket, one requires the bracket $[\cdot, \cdot]_{E}$ to satisfy a Leibniz rule. Denoting functions by $f \in \mathcal{C}^{\infty}(M)$ and sections of $E$ by $s_{i}$, this reads

$$
\left[s_{1}, f s_{2}\right]_{E}=f\left[s_{1}, s_{2}\right]_{E}+\rho\left(s_{1}\right)(f) s_{2},
$$

where $\rho\left(s_{1}\right)$ is a vector field which acts on $f$ as a derivation. If in addition the bracket $[\cdot, \cdot]_{E}$ satisfies a Jacobi identity

$$
\left[s_{1},\left[s_{2}, s_{3}\right]_{E}\right]_{E}=\left[\left[s_{1}, s_{2}\right]_{E}, s_{3}\right]_{E}+\left[s_{2},\left[s_{1}, s_{3}\right]_{E}\right]_{E},
$$

then $\left(E,[\cdot, \cdot]_{E}, \rho\right)$ is called a Lie algebroid.

Moreover, any Lie algebroid can be equipped with a nilpotent exterior derivative as follows

$$
\begin{aligned}
d_{E} \theta^{*}\left(s_{0}, \ldots, s_{n}\right)= & \sum_{i=0}^{n}(-1)^{i} \rho\left(s_{i}\right) \theta^{*}\left(s_{0}, \ldots, \hat{s_{i}}, \ldots, s_{n}\right) \\
& +\sum_{i<j}(-1)^{i+j} \theta^{*}\left(\left[s_{i}, s_{j}\right]_{E}, s_{0}, \ldots, \hat{s}_{i}, \ldots, \hat{s_{j}}, \ldots, s_{n}\right),
\end{aligned}
$$

where $\theta^{*} \in \Gamma\left(\Lambda^{n} E^{*}\right)$ is the analog of an $n$-form on the Lie algebroid and $\hat{s}_{i}$ denotes the omission of that entry. The Jacobi identity of the bracket $[\cdot, \cdot]_{E}$ implies that (C.3) satisfies $\left(d_{E}\right)^{2}=0$. The anchor property and the corresponding formula for the de Rahm differential allow to compute

$$
\begin{aligned}
\left(\left(\Lambda^{n+1} \rho^{*}\right)\left(d_{E} \theta^{*}\right)\right)\left(X_{0}, \ldots, X_{n}\right) & =\left(d_{E} \theta^{*}\right)\left(\rho^{-1}\left(X_{0}\right), \ldots, \rho^{-1}\left(X_{n}\right)\right) \\
& =d\left(\left(\Lambda^{n} \rho^{*}\right)\left(\theta^{*}\right)\right)\left(X_{0}, \ldots, X_{n}\right)
\end{aligned}
$$

with the dual anchor $\rho^{*}=\left(\rho^{t}\right)^{-1}$ and for sections $X_{i} \in \Gamma(T M)$. The relation (C.4) describes how exact terms translate in general.

Open Access. This article is distributed under the terms of the Creative Commons Attribution License (CC-BY 4.0), which permits any use, distribution and reproduction in any medium, provided the original author(s) and source are credited.

\section{References}

[1] A. Dabholkar and C. Hull, Duality twists, orbifolds and fluxes, JHEP 09 (2003) 054 [hep-th/0210209] [INSPIRE].

[2] C.M. Hull, A geometry for non-geometric string backgrounds, JHEP 10 (2005) 065 [hep-th/0406102] [INSPIRE].

[3] J. Shelton, W. Taylor and B. Wecht, Nongeometric flux compactifications, JHEP 10 (2005) 085 [hep-th/0508133] [INSPIRE].

[4] A. Dabholkar and C. Hull, Generalised T-duality and non-geometric backgrounds, JHEP 05 (2006) 009 [hep-th/0512005] [INSPIRE].

[5] T.H. Buscher, A symmetry of the string background field equations, Phys. Lett. B 194 (1987) 59 [INSPIRE]. 
[6] T.H. Buscher, Path integral derivation of quantum duality in nonlinear $\sigma$-models, Phys. Lett. B 201 (1988) 466 [INSPIRE].

[7] N. Hitchin, Generalized Calabi-Yau manifolds, Quart. J. Math. Oxford Ser. 54 (2003) 281 [math/0209099] [INSPIRE].

[8] M. Gualtieri, Generalized complex geometry, math/0401221 [INSPIRE].

[9] M. Graña, R. Minasian, M. Petrini and D. Waldram, T-duality, generalized geometry and non-geometric backgrounds, JHEP 04 (2009) 075 [arXiv:0807.4527] [INSPIRE].

[10] A. Coimbra, C. Strickland-Constable and D. Waldram, Supergravity as generalised geometry I: type II theories, JHEP 11 (2011) 091 [arXiv: 1107.1733] [INSPIRE].

[11] W. Siegel, Two vierbein formalism for string inspired axionic gravity, Phys. Rev. D 47 (1993) 5453 [hep-th/9302036] [INSPIRE].

[12] W. Siegel, Superspace duality in low-energy superstrings, Phys. Rev. D 48 (1993) 2826 [hep-th/9305073] [INSPIRE].

[13] O. Hohm and S.K. Kwak, Frame-like geometry of double field theory, J. Phys. A 44 (2011) 085404 [arXiv: 1011.4101] [INSPIRE].

[14] D. Geissbuhler, D. Marques, C. Núñez and V. Penas, Exploring double field theory, JHEP 06 (2013) 101 [arXiv:1304.1472] [INSPIRE].

[15] C. Hull and B. Zwiebach, Double field theory, JHEP 09 (2009) 099 [arXiv:0904.4664] [INSPIRE].

[16] O. Hohm, C. Hull and B. Zwiebach, Background independent action for double field theory, JHEP 07 (2010) 016 [arXiv: 1003.5027] [INSPIRE].

[17] O. Hohm, C. Hull and B. Zwiebach, Generalized metric formulation of double field theory, JHEP 08 (2010) 008 [arXiv: 1006.4823] [INSPIRE].

[18] G. Aldazabal, D. Marques and C. Núñez, Double field theory: a pedagogical review, Class. Quant. Grav. 30 (2013) 163001 [arXiv:1305.1907] [INSPIRE].

[19] D.S. Berman and D.C. Thompson, Duality symmetric string and M-theory, arXiv: 1306.2643 [INSPIRE].

[20] O. Hohm, D. Lüst and B. Zwiebach, The spacetime of double field theory: review, remarks and outlook, Fortsch. Phys. 61 (2013) 926 [arXiv:1309.2977] [INSPIRE].

[21] D. Andriot, M. Larfors, D. Lüst and P. Patalong, A ten-dimensional action for non-geometric fluxes, JHEP 09 (2011) 134 [arXiv:1106.4015] [INSPIRE].

[22] D. Andriot, O. Hohm, M. Larfors, D. Lüst and P. Patalong, Non-geometric fluxes in supergravity and double field theory, Fortsch. Phys. 60 (2012) 1150 [arXiv:1204.1979] [INSPIRE].

[23] D. Andriot and A. Betz, $\beta$-supergravity: a ten-dimensional theory with non-geometric fluxes and its geometric framework, JHEP 12 (2013) 083 [arXiv:1306.4381] [INSPIRE].

[24] R. Blumenhagen, A. Deser, E. Plauschinn and F. Rennecke, Non-geometric strings, symplectic gravity and differential geometry of Lie algebroids, JHEP 02 (2013) 122 [arXiv:1211.0030] [INSPIRE]. 
[25] R. Blumenhagen, A. Deser, E. Plauschinn, F. Rennecke and C. Schmid, The intriguing structure of non-geometric frames in string theory, Fortsch. Phys. 61 (2013) 893 [arXiv: 1304.2784] [INSPIRE].

[26] D. Andriot and A. Betz, NS-branes, source corrected Bianchi identities and more on backgrounds with non-geometric fluxes, JHEP 07 (2014) 059 [arXiv:1402.5972] [INSPIRE].

[27] R.A. Reid-Edwards and B. Spanjaard, $N=4$ gauged supergravity from duality-twist compactifications of string theory, JHEP 12 (2008) 052 [arXiv:0810.4699] [INSPIRE].

[28] O. Hohm and S.K. Kwak, Double field theory formulation of heterotic strings, JHEP 06 (2011) 096 [arXiv:1103.2136] [INSPIRE].

[29] D. Andriot, Heterotic string from a higher dimensional perspective, Nucl. Phys. B $\mathbf{8 5 5}$ (2012) 222 [arXiv:1102.1434] [INSPIRE].

[30] M. Garcia-Fernandez, Torsion-free generalized connections and heterotic supergravity, Commun. Math. Phys. 332 (2014) 89 [arXiv:1304.4294] [INSPIRE].

[31] D. Baraglia and P. Hekmati, Transitive Courant algebroids, string structures and T-duality, arXiv: 1308.5159 [INSPIRE].

[32] L.B. Anderson, J. Gray and E. Sharpe, Algebroids, heterotic moduli spaces and the Strominger system, JHEP 07 (2014) 037 [arXiv:1402.1532] [INSPIRE].

[33] X. de la Ossa and E.E. Svanes, Holomorphic bundles and the moduli space of $N=1$ supersymmetric heterotic compactifications, JHEP 10 (2014) 123 [arXiv:1402.1725] [INSPIRE].

[34] M. Graña and D. Marques, Gauged double field theory, JHEP 04 (2012) 020 [arXiv: 1201.2924] [INSPIRE].

[35] O.A. Bedoya, D. Marques and C. Núñez, Heterotic $\alpha^{\prime}$-corrections in double field theory, JHEP 12 (2014) 074 [arXiv: 1407.0365] [INSPIRE].

[36] A. Coimbra, R. Minasian, H. Triendl and D. Waldram, Generalised geometry for string corrections, JHEP 11 (2014) 160 [arXiv:1407.7542] [INSPIRE].

[37] X. de la Ossa and E.E. Svanes, Connections, field redefinitions and heterotic supergravity, JHEP 12 (2014) 008 [arXiv: 1409.3347] [INSPIRE].

[38] O. Hohm, W. Siegel and B. Zwiebach, Doubled $\alpha^{\prime}$-geometry, JHEP 02 (2014) 065 [arXiv: 1306.2970] [INSPIRE].

[39] O. Hohm and B. Zwiebach, Green-Schwarz mechanism and $\alpha^{\prime}$-deformed Courant brackets, JHEP 01 (2015) 012 [arXiv: 1407.0708] [INSPIRE].

[40] O. Hohm and B. Zwiebach, Double field theory at order $\alpha^{\prime}$, JHEP 11 (2014) 075 [arXiv: 1407.3803] [INSPIRE].

[41] E. Bergshoeff, I. Entrop and R. Kallosh, Exact duality in string effective action, Phys. Rev. D 49 (1994) 6663 [hep-th/9401025] [INSPIRE].

[42] M. Serone and M. Trapletti, A note on T-duality in heterotic string theory, Phys. Lett. B 637 (2006) 331 [hep-th/0512272] [INSPIRE].

[43] R. Blumenhagen, A course on noncommutative geometry in string theory, Fortsch. Phys. 62 (2014) 709 [arXiv:1403.4805] [INSPIRE]. 
[44] G. Aldazabal, W. Baron, D. Marques and C. Núnez, The effective action of double field theory, JHEP 11 (2011) 052 [Erratum ibid. 11 (2011) 109] [arXiv:1109.0290] [INSPIRE].

[45] R. Blumenhagen, X. Gao, D. Herschmann and P. Shukla, Dimensional oxidation of non-geometric fluxes in type II orientifolds, JHEP 10 (2013) 201 [arXiv:1306.2761] [INSPIRE].

[46] P. Bouwknegt, K. Hannabuss and V. Mathai, Nonassociative tori and applications to T-duality, Commun. Math. Phys. 264 (2006) 41 [hep-th/0412092] [INSPIRE].

[47] R. Blumenhagen and E. Plauschinn, Nonassociative gravity in string theory?, J. Phys. A 44 (2011) 015401 [arXiv: 1010.1263] [INSPIRE].

[48] D. Lüst, T-duality and closed string non-commutative (doubled) geometry, JHEP 12 (2010) 084 [arXiv: 1010.1361] [INSPIRE].

[49] R. Blumenhagen, A. Deser, D. Lüst, E. Plauschinn and F. Rennecke, Non-geometric fluxes, asymmetric strings and nonassociative geometry, J. Phys. A 44 (2011) 385401 [arXiv:1106.0316] [INSPIRE].

[50] D. Mylonas, P. Schupp and R.J. Szabo, Membrane $\sigma$-models and quantization of non-geometric flux backgrounds, JHEP 09 (2012) 012 [arXiv: 1207.0926] [INSPIRE].

[51] R. Blumenhagen, M. Fuchs, F. Haßler, D. Lüst and R. Sun, Non-associative deformations of geometry in double field theory, JHEP 04 (2014) 141 [arXiv: 1312.0719] [INSPIRE].

[52] J. Polchinski and E. Witten, Evidence for heterotic type-I string duality, Nucl. Phys. B 460 (1996) 525 [hep-th/9510169] [INSPIRE]. 